undereating, and is a physiological perpetuating factor of anorexia nervosa (Robinson, Journal, March $1989,154,400-405)$. In the study of Stacher et al where gastric symptoms were specifically measured, it is noteworthy that all the anorexic patients showed varying degrees of gastric fullness, pain, belching, and bloating.

In those Asian cultures where a permeative cultural fear of fatness is inconspicuous, stomach affliction may be a more admissible means of adopting the sick role and negotiating change in interpersonal worlds than complaints of fatness or depression. Among Hong Kong Chinese, we see a phenomenological mixture of anorexic patients, some of whom used epigastric bloating and/or pain rather than the fear of fatness to legitimate food refusal and emaciation (Lee, 1991). Delayed gastric emptying may conceivably contribute to their culturally amplified gastric complaint, which should not be presumed to be a purely psychological defense. Stacher $e t$ al have shown that the enhancement of gastric emptying may be therapeutically useful, and perhaps more meaningful than confronting anorexic patients about their body image distortion. If the finding can be substantiated in the long-term treatment of a larger group of anorexic patients, the implications for the often difficult treatment of both Western and non-Western anorexic patients may be considerable.

Casper, R. C. (1983) On the emergence of bulimia nervosa as a syndrome: a historical view. International Journal of Eating Disorders, 2, 3-16.

Lee, S. (1991) Anorexia nervosa in Hong Kong-a Chinese perspective. Psychological Medicine, 21, 703-711.

PARRY-JONES, B. (1991) Historical terminology of eating disorders. Psychological Medicine, 21, 21-28.

SHORTER, E. (1987) The first great increase in anorexia nervosa. Journal of Social History, 21, 69-96.

Department of Psychiatry

SING LeE

Chinese University of Hong Kong

Prince of Wales Hospital

Shatin, Hong Kong

\section{Blood-letting in bulimia nervosa}

SIR: Parkin \& Eagles (Journal, February 1993, 162, 246-248) reported three cases of blood-letting in association with bulimia nervosa, and noted this association had not previously been described. We would like to report a similar case.

Case report. Our patient, in her 20 s, described an uneventful early childhood but recalled lifelong difficulty socialising, associated with lack of self-confidence. She did well academically at university, but had a limited social life with no sexual relationships. She had a period of in-patient treat- ment eight years ago for anorexia nervosa and was treated as an out-patient two years ago following episodes of selfmutilation. There was a positive family history, her younger sister having been treated for anorexia nervosa and trichotillomania.

She was referred, on this occasion, because of episodes of self-mutilation, blood-letting, bingeing, and depressed mood. She described cutting herself, after which she sutured the wound without anaesthetic. On one occasion she sutured a self-inflicted cut on her leg while engaged in a telephone conversation. She also described two episodes of performing venisection on herself. On each occasion she withdrew approximately a pint and a half of blood which she then poured down the sink. She described excessive intake of alcohol of 60 units per week, and depressive symptoms which met DSM - III - R criteria for dysthymic disorder. She had previously met DSM - III - R criteria for bulimia nervosa, although she currently did not. She weighed $57.2 \mathrm{~kg}$ and her height was $1.7 \mathrm{~m}$.

She was on fluoxetine, $20 \mathrm{mg}$ daily, and this was increased to $60 \mathrm{mg}$ daily. She was also referred to a Day Hospital Group for assertiveness training. Less than one month later she required admission to the general hospital having venisected herself again. Her haemoglobin was $4.6 \mathrm{~g} / \mathrm{l}$ and she required a blood transfusion.

Three months after presentation she was euthymic, she had returned to work, and she felt no desire to cut or venisect herself. Her eating pattern was normal and she had successfully completed assertiveness training. She remained on fluoxetine, $60 \mathrm{mg}$ daily. She reported that she had not obtained any relief from cutting or venisecting. Her cutting had started two years earlier and although initially this relieved tension, it no longer did. She was unable to identify any urge to venisect, and after venisecting she described feeling 'numb'. The venisection had not been accompanied by any suicidal intent or performed in the hope of weight loss.

There are a number of similarities between this case and the three previously described. Our patient is a veterinary surgeon with access to instruments, and has knowledge of blood-letting. She had previously engaged in acts of self-harm, and in the past had met DSM - III - R criteria for bulimia nervosa. However, a notable difference is the fact that this patient does not derive any apparent psychological benefit from blood-letting and was 'mystified' as to why she did it.

\section{Bellsdyke Hospital Bellsdyke Road Lambert}

Abuse of the nasogastric tube in patients with eating disorders

SIR: The case reports by Parkin \& Eagles (Journal, February 1993, 162, 246-248) describing bloodletting in bulimia nervosa highlight the self-abusive 